\title{
BASE PEDAGÓGICA PARA GENERAR UN MODELO NO FORMAL DE EDUCACIÓN AMBIENTAL
}

\author{
Jorge Villadiego-Lorduy \\ Dennis Huffman-Schwocho $\mathbb{B D}^{2}$ \\ Stalyn Guerrero Gómez (1D ${ }^{3}$ \\ Adolfo Cortecero-Bossio (D) 4
}

Recibido el 7 de septiembre de 2015, aprobado el 12 de mayo de 2016 y actualizado el 28 de diciembre de 2016

DOI: 10.17151/luaz.2017.44.19

\section{Resumen}

En el presente escrito se busca establecer la base pedagógica más acorde para la generación de un modelo de educación no formal para la protección de los humedales de Bañó y los Negros, basándose en estudios previos de valoración ambiental e identificación de los factores que están incidiendo en conductas ambientalmente negativas al medio. Se define como elementos pedagógicos a considerar el aprendizaje significativo, la andragogía y el enfoque sistémico de la enseñanza. Se desarrolla una conceptualización de la educación ambiental en sus diferentes niveles: formal, no formal e informal. Se parte del aprendizaje significativo y la necesidad de relacionar nuevos conceptos con conocimientos previos, se resaltan sus principales características y se aclara lo que no se considera un aprendizaje significativo. Por otra parte, en cuanto a la educación de adultos se refiere o andragogía, se establecen algunas definiciones para la misma y sus principios fundamentales: horizontalidad y participación. Se explica cómo a través de la andragogía, el adulto aprende a valorar las experiencias propias y ajenas en función de la construcción de un proyecto de vida. Se indica además que el proceso educativo desde la andragogía debe ser personalizado e implica un auto aprendizaje donde el individuo es el único responsable del mismo. También se enfatiza cómo, desde la teoría sistémica de la enseñanza, se puede obtener una percepción de la realidad desde diferentes puntos de vista, evitando parcelaciones o sesgos ante distintos saberes o disciplinas, con el objetivo de poder establecer las diferentes interrelaciones que puedan darse para determinada(s) realidad(es). Por último, se sustenta cómo podría aplicarse tanto el aprendizaje significativo, la andragogía y la teoría sistémica de la enseñanza en la generación del modelo educativo, además de realizar las respectivas conclusiones y recomendaciones frente al tema.

\section{Palabras clave}

Modelos educativos, conocimientos previos, participación y horizontalidad.

\section{PEDAGOGICAL BASIS FOR GENERATING A NON-FORMAL ENVIRONMENTAL EDUCATIONAL MODEL}

\begin{abstract}
The purpose of this article is to establish the most appropriate pedagogical basis for the formulation of a non-formal educational model for the protection of the Bañó and Los Negros wetlands, using previous studies on environmental assessment and identification of the factors which influence negative environmental conduct. Meaningful learning, andragogy and the systemic focus for teaching are defined as pedagogical
\end{abstract}


elements. A conceptualization of environmental education in its different levels -formal, non-formal and informal- were also developed. From significant learning and the need to relate new concepts to previous knowledge the main characteristics were highlighted and it was clarified what was not considered meaningful learning. Moreover, in terms of adult education or andragogy, some definitions were established along with its fundamental principles: horizontal alignment and participation. It is explained how through andragogy adults learn to appreciate their own and others experiences around the construction of life projects. It is also indicated that the educational process from andragogy should be personalized implying self-learning in which the individual is the only one responsible for it. It also emphasizes how, through the systemic theory of teaching, it is possible to obtain a perception of reality from different points of view avoiding fragmentations or bias concerning different knowledge or disciplines in order to establish different interrelations that could occur in certain realities. Finally, how significant learning, andragogy and the systemic theory of teaching could be used in the formulation of an educational model is discussed, along with drawing the respective conclusions and recommendations concerning this matter.

\section{Key words}

Educational models, prior knowledge, participation and horizontal alignment

\section{MATERIALES Y METODOS}

La metodología implementada en esta investigación fue de carácter explicativo en la cual se buscó, en un primer momento, analizar una serie de teorías educativas (aprendizaje significativo, andragogía, teoría sistémica de la enseñanza, entre otras) que sirvieran de base pedagógica para un modelo no formal de educación ambiental, que contribuyera a la conservación con fines de protección de las Ciénagas de Bañó y Los Negros en el departamento de Córdoba, Colombia. En un segundo momento, se planteó interpretar cómo se relacionaban los diferentes factores socio-ambientales de las comunidades cercanas a dichos humedales con las teorías pedagógicas expuestas, con el fin de sustentar la interrelación de diferentes elementos pedagógicos que pudieran justificar un modelo educativo no formal de carácter participativo que respondiera a un contexto en particular, por ultimo. En un tercer instante, se concluyó e hicieron las recomendaciones necesarias sobre toda la temática desarrollada.

Del mismo modo, los métodos de investigación que se utilizaron para determinar la base pedagógica más acorde para la generación de dicho modelo educativo se soportó, por una parte, en el análisis de contenido a profundidad tanto de información primaria como secundaria; y en segunda instancia, en la implementación de métodos de estadística inferencial, basado en el cálculo de estimaciones porcentuales y valores absolutos. Para el caso de la información secundaria, se compiló información que permitió establecer aportaciones teóricas (estudio de teorías educativas), con el propósito de identificar elementos pedagógicos que sirvieran para estructurar el modelo. Una vez finalizada esta parte del análisis teórico, se cruzó esto con los datos estadísticos obtenidos de la aplicación de un instrumento de investigación tipo cuestionario, que buscó recopilar información de variables socioeconómicas, de problemas y conflictos ambientales y de percepción y cultura ambiental de los habitantes del área de interés. La población universo de la investigación estuvo determinada por 645 viviendas habitadas pertenecientes a los corregimientos de Cotocá Arriba (110), Castilleral (146) y Palo de Agua (389). Se determinó un diseño de muestreo por estrato, donde los estratos corresponden a los diferentes pueblos, para Cotocá Arriba y Castilleral se les aplicó un muestreo sistemático y en el caso de Palo 
de Agua se desarrolló un muestreo aleatorio simple. Se aplicó un total de 72 encuestas, distribuidas de la siguiente forma: Cotocá Arriba y Castilleral, 12 cada una, Palo de Agua 48. El software con el que se trabajó el análisis estadístico fue R versión 3.0.2.

\section{Introducción}

La teoría del aprendizaje significativo fue desarrollada por David Ausubel hacia la década de 1970 y se basa en la asociación de los conocimientos previos que tiene el individuo con los conocimientos nuevos que va adquiriendo, tal asociación forma el nuevo aprendizaje. El aprendizaje significativo busca darle un uso a lo aprendido en nuevas situaciones, además de la integración y articulación de significados y que el aprendizaje adquirido sea a largo plazo y produzca un cambio cognitivo. Por otro lado, el término andragogía fue originado por Alexander Kapp en 1833, refiere a la teoría y la práctica de la educación de adultos y existe como concepto formal a partir del siglo XIX, teniendo reconocimiento mundial durante este siglo. Tiene dos principios básicos: la horizontalidad y la participación. El auto aprendizaje es clave en el desarrollo de procesos educativos bajo un enfoque andragógico y más que buscar una formación en éste o los individuos, lo que pretende es la generación de proyectos de vida. En la andragogía, el adulto decide qué aprender y es él mismo quien traza los objetivos y metas a alcanzar. Así mismo, la teoría sistémica de la enseñanza promovida por Robert Gagné y la cual es una adaptación de la teoría general de sistemas desarrollada por Ludwig von Bertalanffy (1930), busca generar procesos de instrucción enmarcados en una totalidad a partir de la transferencia de conocimientos desde varios campos de la enseñanza, que permita la adquisición de saberes a través de una estructura lógica y sistemática.

Lo interesante de establecer una base pedagógica enmarcada en el aprendizaje significativo, la andragogía y la teoría sistémica de la enseñanza para la generación de un modelo no formal de educación ambiental, radica en que éstos proporcionan suficientes elementos para trabajar con una población campesina adulta y facilitan la adquisición de nuevos conocimientos a largo plazo, a partir de procesos eficientes de aprendizaje, como también a fomentar la participación y compromiso de los individuos que integran las comunidades aledañas a los espejos de agua y la construcción desde la individualidad hacia la colectividad de proyectos de vida que, en su sumatoria, podrían convertirse escenarios viables para mejorar la relación ser humano-medio y poder contribuir al desarrollo ambiental del territorio.

\section{La educación ambiental (EA): formal, no formal e informal}

La educación ambiental se sitúa en los años 70, aunque el término educación ambiental ya aparece en documentos de la UNESCO (Organización de las Naciones Unidas para la Ciencia, la Educación y la Cultura), datados de 1965; sin embargo, no es hasta el año 1972, en Estocolmo, durante la conferencia de las Naciones Unidas sobre el medio humano, cuando se reconoce oficialmente la existencia de este concepto y de su importancia para cambiar los modelos de desarrollo. Hoy día, la EA se ha convertido en un poderoso instrumento de la gestión ambiental y debe entenderse como un proceso dinámico y participativo, que busca despertar en la población una conciencia que le permita identificarse con la problemática ambiental general y específica. Guier (2002, pp.2-3) define la EA como "un proceso inherente a todo espacio educativo, conducente a la formación de personas despiertas a su 
realidad y conscientes de que pertenecen a un sistema ecológico global, regido por una serie de leyes y principios que deben conocerse y sobe todo, respetarse para garantizar la vida misma". Adicionalmente, la educación ambiental se divide en tres categorías: formal, no formal e informal. La primera, inmersa en el sistema educativo tradicional y organizado en un currículo de carácter transversal. La segunda, fundamentada en la transmisión de conocimientos, aptitudes y valores ambientales fuera del sistema educativo tradicional y que conllevan a que las personas beneficiarias de este tipo de educación, desarrollen aptitudes positivas con el ambiente; su fin no es alcanzar certificaciones o títulos. La última, y no menos importante, se da de forma espontánea y no planificada, no responde a estructuras pedagógicas. Generalmente, cuando se habla de educación, lo primero en lo que se piensa es en la escuela. La educación ha sido limitada a escolaridad y desgraciadamente se descarta o se resta importancia a otras prácticas, espacios y escenarios sociales que son tanto o más importantes para la formación de las personas (Buenfil et al, 1991). Para González (1993) la educación no formal (ENF) es aquélla que se desarrolla paralela o independiente a la educación formal y que por tanto, no queda inscrita en los programas del ciclo del sistema escolar y aunque las experiencias educativas sean secuenciales, no se acredita y no se certifica. En 1974, Coombs y Ahmed (citados por Camors, 2009, p.24) proponen la distinción conceptual entre educación formal, no formal e informal. Llamaban educación no formal a "toda actividad organizada, sistemática, educativa, realizada fuera del marco del sistema oficial, para facilitar determinadas clases de aprendizajes a subgrupos particulares de la población, tanto adultos como niños". En el mismo sentido, Trilla (1996) afirma que la educación no formal es una modalidad de la educación. El concepto surge históricamente ante los problemas y dificultades del desarrollo de la escolarización, como una forma de alcanzar los objetivos educativos propuestos para toda la población. Surge precisamente para completar, reforzar, o continuar, en su caso, supliendo ciertos cometidos escolares.

\section{Aprendizaje significativo}

Para hablar de aprendizaje significativo primero hay que comprender qué se entiende por aprendizaje y los tipos de aprendizaje existentes. El aprendizaje es un proceso mediante el cual hay adquisición de conocimientos, habilidades, valores y actitudes, ya sea a través de la enseñanza, el estudio o la experiencia. Existen básicamente seis tipos de aprendizaje que van desde el aprendizaje receptivo, que se basa en la comprensión o entendimiento de un contenido con el fin de poder reproducirlo; pasando por el aprendizaje mecanicista o repetitivo, que ocurre cuando el individuo memoriza contenidos sin comprenderlos. El aprendizaje por descubrimiento se soporta en el descubrimiento de conceptos y sus relaciones para reordenarlos y adaptarlos al esquema cognitivo de un individuo en particular. Cuando se observa a una persona que sirve como modelo, se está hablando de un aprendizaje observacional; así mismo, en el aprendizaje latente el educando obtiene un nuevo conocimiento, pero es necesario que se ofrezca un incentivo para que éste lo muestre; por último, se encuentra el aprendizaje significativo que relaciona conocimientos viejos con nuevos.

Un aprendizaje es significativo cuando los contenidos son relacionados de modo no arbitrario y sustancial (no al pie de la letra) con lo que el alumno ya sabe. "Por relación sustancial y no arbitraria se debe entender que las ideas se relacionan con algún aspecto existente específicamente relevante de la estructura cognoscitiva del alumno, como una imagen, un símbolo ya significativo, un concepto o una proposición" (Ausubel et al, 1983, p.18). El mismo autor señala, que el aprendizaje significativo ocurre cuando una nueva información "se conecta" con un concepto relevante ("subsunsor") preexistente en la estructura cognitiva, lo que implica que, las nuevas ideas, conceptos y proposiciones pueden ser aprendidos significativamente en la 
medida en que otras ideas, conceptos o proposiciones relevantes estén adecuadamente claras y disponibles en la estructura cognitiva del individuo y que funcionen como un punto de "anclaje" a las primeras. Por lo tanto, el aprendizaje significativo, como lo señala Ausubel, está asociado a la construcción de un nuevo conocimiento a partir de ideas previas por parte del individuo. Así mismo, es necesaria la interacción que se genere entre el nuevo conocimiento adquirido y la estructura de conocimiento existente, es decir, la estructura cognitiva que posea la persona, de tal forma que éste adquiere un significado y se integre a la misma. Por último, los conceptos en el aprendizaje significativo deben ir de lo general a lo particular, con el fin de dar origen a un aprendizaje a largo plazo, no generando un conocimiento memorístico cortoplacista, sino tratando de estructurar una red entre los conceptos ya adquiridos con los nuevos. Para Rodríguez (2008, p.26), "No se genera aprendizaje significativo si no están presentes las ideas de anclaje pertinentes en la estructura cognitiva del aprendiz. Es un requisito indispensable sin el cual no hay modo de enlazar las nuevas informaciones con las existentes en la mente de los sujetos".

De igual forma, un aprendizaje significativo se desarrolla cuando el material de aprendizaje utilizado por el educador pueda relacionarse de forma lógica y sustancial, con alguna estructura cognitiva que posea el individuo. Así mismo, cuando el significado potencial entendido como adquisición de un contenido cognoscitivo nuevo por parte del individuo se convierte en significado psicológico dependiendo de dos aspectos fundamentales: el primero, de la representación que el alumno haga del material lógicamente significativo, y segundo, de las ideas que posea con anterioridad en su estructura cognitiva. No menos importante, se señala la disposición del individuo para el aprendizaje significativo, es decir, la capacidad de relacionar el nuevo conocimiento con su estructura cognitiva (VerCuadro 1). Cabe señalar que dentro de las herramientas utilizadas para desarrollar procesos de aprendizaje significativo se tiene a los mapas conceptuales (MC). Los MC son esquemas para la representación del conocimiento mediante los cuales se hacen evidentes, tanto los conceptos como la forma en que se enlazan éstos para formar proposiciones. Constituyen redes en las que los nodos son los conceptos y los enlaces contienen las palabras que relacionan a los conceptos (Ojeda et al, 2007). Mediante MC se pueden entrelazar conceptos y crear redes que permiten captar lo más significativo de un tema.

\section{Cuadro 1. Condiciones para el logro del aprendizaje significativo}

\begin{tabular}{|c|l|}
\hline \multicolumn{2}{|l|}{ Respecto al } \\
\hline \multirow{2}{*}{ a) Material } & Relacionabilidad no arbitraria \\
\cline { 2 - 2 } & Relacionabilidad sustancial \\
\cline { 2 - 2 } & $\begin{array}{l}\text { Estructura y organiza ción (Significado } \\
\text { lógico) }\end{array}$ \\
\cline { 2 - 2 } & \\
\hline b) Ahunmo & Disposición o actitud \\
\cline { 2 - 2 } & $\begin{array}{l}\text { Naturaleza de su estructura cognitiva } \\
\text { Conocimientos y experiencias previas } \\
\text { (Significado psicológico) }\end{array}$ \\
\hline
\end{tabular}

Fuente: Díaz, F. Estrategias docentes para un aprendizaje significativo.

Para Bolívar (2009, p.2),

Un aprendizaje basado en la repetición tiende a inhibir el nuevo aprendizaje, mientras que el aprendizaje significativo facilita el nuevo aprendizaje 
relacionándolo. Los aprendizajes por repetición son entidades desconectadas, aisladas y dispersas en la mente del alumno, son aprendizajes de rápido olvido que permiten una repetición inmediata y cierta, pero que no son aprendizajes en sí, porque es un aprendizaje que no es real y por tanto no es significativo. El ser humano tiene la disposición de aprender, de verdad, sólo aquello a lo que le encuentra sentido o lógica.

Además, "El ser humano tiende a rechazar aquello a lo que no le encuentra sentido. El único auténtico aprendizaje es el aprendizaje significativo, el aprendizaje con sentido. Cualquier otro aprendizaje será puramente mecánico, memorístico, coyuntural: aprendizaje para aprobar un examen, para ganar la materia, etc". El aprendizaje significativo es un aprendizaje relacional. El sentido lo da la relación del nuevo conocimiento con conocimientos anteriores, con situaciones cotidianas, con la propia experiencia, con situaciones reales, etc". Aprender significativamente requiere también una actitud crítica de cuestionamiento que favorezca la toma de decisiones y posibilite el planteamiento de preguntas relevantes (Moreira, 2000b). En el mismo orden de ideas, el aprendizaje significativo presenta tres tipos de aprendizaje: A) El aprendizaje de representaciones consiste en dar significado a determinados símbolos; B) El aprendizaje de conceptos se enmarca en la definición de los conceptos como objetos, eventos, situaciones que poseen atributos comunes y que se designan mediante un símbolo; y C) El aprendizaje de proposiciones que implica la relación y combinación de palabras, donde cada una se convierte en un referente unitario con el fin de generar una idea y un nuevo significado que son asimilados por la estructura cognoscitiva. Así mismo, el aprendizaje significativo tiene una serie de ventajas que van desde la retención más duradera de la información, la facilidad de relacionar conocimientos nuevos con anteriores, hasta el desarrollo personal y activo por parte del individuo.

\section{Andragogía}

La andragogía es el arte de la educación de los adultos, según Knowles (1980) la andragogía es el arte y la ciencia de ayudar a adultos a aprender. Fernández (2001) la define como la disciplina que se ocupa de la educación y aprendizaje del adulto. Para Rojano, (2008) la andragogía es ciencia, arte o disciplina relacionada con el aprendizaje permanente de personas adultas, donde hay un adulto que guía el proceso y otro adulto interesado en modificar, aumentar $u$ obtener cierto aprendizaje voluntariamente. Aquí, el adulto se torna en un ser que auto dirige su aprendizaje, aprovecha sus experiencias para lograrlo, mostrándose analítico y crítico de las acciones tratadas en clases y bajo ciertas condiciones psicológicas y ambientales del momento. Por lo general, los autores que se dedican a estudiar andragogía aceptan como características del adulto, entre otras, las siguientes: Persona capaz de crear, de asumir con entereza responsabilidades en torno a ciertos asuntos inherentes a la vida social y de tomar decisiones con plena libertad (Alcalá, 2001). El mismo autor afirma que la andragogía proporciona la oportunidad para que el adulto que decide aprender, participe activamente en su propio aprendizaje e intervenga en la planificación, programación, realización y evaluación de las actividades educativas en condiciones de igualdad con sus compañeros, participantes y con el facilitador. Lo anterior, conjuntamente con un ambiente de aprendizaje adecuado, determina lo que podría llamarse una buena praxis andragógica ${ }^{5}$. El adulto construye saberes para orientarse en situaciones de incertidumbre es una persona en proceso de humanización creciente que conlleva un despliegue de creatividad (Ludojoski, 2008). Tratar con el adulto es moverse entre historias comunitarias, locales, nacionales, regionales y mundiales, e historias personales de vida entrelazadas, que conforman tramas desde lo cotidiano (Valdez, 2011). 
Cabe señalar que la andragogía presenta dos principios fundamentales basados en la horizontalidad y la participación. La primera, puede entenderse como una relación entre iguales y más explícitamente como una relación compartida de actitudes y de compromisos hacia logros y resultados exitosos. Es una relación entre iguales que se entienden mutuamente, con una comprensión y un entendimiento que generan compromiso por metas comunes y objetivos individuales que no son vistos como "del otro" o "ajenos", sino propios, míos o nuestros. Por dichos objetivos, un individuo va a trabajar y ayudar a que "el otro" llegue hasta donde sus potencialidades se lo permitan (Torres et al; 1994). La segunda, según Adam (1971), se define como la acción de tomar decisiones en conjunto o tomar parte con otros en la ejecución de una tarea determinada. La participación es el acto de compartir algo: es un dar y recibir, involucrarse en un proyecto común. Es buscar explicaciones a los indicios de temor, desconfianza, incomunicación, competencia. Revisar con frecuencia las metas, adaptarlas o cambiarlas. Oír sugestiones, compartir liderazgo, tomar en cuenta motivaciones y capacidades personales; aportar. La andragogía constituye a la vez un enfoque, en el sentido de proporcionar cierta óptica para mirar, nombrar y teorizar en torno al hecho educativo, y una estrategia, en el sentido de presentar conceptos, principios y lineamientos para orientar la vivencia de los adultos en procesos de aprendizaje (Valdez, 2011). La educación andragógica cuenta con una serie de factores que son claves en su desarrollo, partiendo de la relación entre los elementos, el control de procesos de dirección, el énfasis del aprendizaje, los procesos mentales, el planeamiento, administración y evaluación del aprendizaje, y por último, la dirección del proceso (Ver Cuadro 2).

\section{Cuadro 2. Factores del modelo andragógico.}

\begin{tabular}{|c|c|}
\hline FACTORES & MODELO AINDRAGOGICO \\
\hline Relación entre los elementos & Adulto - Adulto. \\
\hline & Horizontal - Participativa. \\
\hline Control de procesos de Dirección & Participante(s). \\
\hline Énf asis del aprendizaje & $\begin{array}{l}\text { En los procesos de aprender y fuentes de } \\
\text { información. }\end{array}$ \\
\hline Procesos mentales & $\begin{array}{l}\text { El pens amiento lógico, los proces os mentales } \\
\text { superiores se hacen presentes. La imaginación } \\
\text { juega un papel excepcional hacia la } \\
\text { creatividad. }\end{array}$ \\
\hline $\begin{array}{l}\text { Planeamiento, administración } \quad y \\
\text { evaluación del aprendizaje }\end{array}$ & $\begin{array}{l}\text { Centrada en los participantes o compartida } \\
\text { con el facilitador. }\end{array}$ \\
\hline Dirección del proceso & Orientación - aprendizaje. \\
\hline
\end{tabular}

Fuente: Tomado de Monografía andragogía y educación a distancia.

Unidad 2 Fac. Enseñanza Dirigida U. H., según libro Andragogia y Docencia Universitaria de Félix Adam y Asociados. Fondo Editorial de la FIDEA. Venezuela.

La andragogía ha de integrar realidades disímiles entre diversos grupos y actores, en un universo de información que lucha entre la identidad y la diferencia, entre la unidad y la pluralidad. Ello la pone frente a la creatividad y la transgresión de lo cotidiano, y a la búsqueda de armonización de los rasgos interdisciplinarios de los participantes (Pereira, sf). En la andragogía, a diferencia de otros modelos o estrategias pedagógicas, el adulto aprende valorando las experiencias propias y ajenas en pro de la construcción de un proyecto de vida, igualmente el proceso la educación debe ser personalizada,, lo que implica que hay un auto aprendizaje y que el individuo es el único responsable del mismo, no indicando lo anterior que se construyan comunidades 
de aprendizaje a partir de objetivos individuales, así como el centro del proceso de aprendizaje es el educando y su deseo de aprender. Para Valdez (2011), el papel del adulto en la andragogía se sintetiza en: A) activos, debido a que son autónomos a la hora de interpretar el mundo y en el manejo de sus emociones; B) con predisposición a la integración de grupos y alianzas sociales; $C$ ) en constantes procesos evolutivos que permiten ver todas sus facetas como ser humano; y D) transforma constantemente las realidades y constituye una síntesis social que reapropia y reproduce lo social. Por lo tanto, los procesos de aprendizaje en la andragogía son proyectos de vida que parten de una iniciativa individual de formación que impacta en el entorno y los transforma y propende por la creación colectiva de un ambiente de aprendizaje, en el cual el auto aprendizaje es el derrotero a seguir con el propósito de generar un proceso de aprendizaje eficiente, y principios como la libertad, el respeto, solidaridad, entre otros que están inmersos en este tipo de educación.

\section{Teoría sistémica de la enseñanza}

La teoría sistémica de la enseñanza resulta de la aplicación de la teoría general de sistemas al campo educativo, y su principal representante es Robert Gagné. Valenzuela (2004) señala que la teoría general de sistemas es un conjunto de definiciones, postulados, hipótesis y proposiciones vinculadas entre sí, que tratan la realidad como una jerarquía integrada de organizaciones de materia y energía, su precursor fue el austriaco L. von Bertalanffy. En el mismo sentido, Antolín (2010) considera que el pensamiento sistémico integral tiene su base en la percepción de la realidad de una visión desde todos los puntos de vista y no sólo de la disciplina o aparente. Es por ello que este enfoque genera un análisis, comprensión y accionar distinto del conocido método científico, que sólo percibe partes de esta realidad y de manera particular, no conectada con un mundo que afecta.

Ahora, desde la educación sistémica, Nemeth (1991) considera unos presupuestos básicos de este tipo de educación, definidos en: A) facilitar al estudiante el aprendizaje de aquello que desea y necesita aprender; B) enseñarle como aprender más eficientemente; y C) motivarle para que aprenda particularmente, aquellas cosas que necesita, en orden de satisfacer sus deseos y curiosidades y que a su vez sean socialmente útiles. Igualmente, el mismo autor recalca que un sistema educacional sistémico debe garantizar la preservación de las diferencias individuales entre los alumnos o estudiantes, la focalización en el aprendizaje motivando el deseo de aprender más; así mismo, orientar al estudiante a aprender a sintetizar porque esto es lo que produce el entendimiento y la habilidad para saber. Dotar al estudiante con la capacidad, animándolo a manejar la realidad como un todo, como un sistema en vez de orientar el análisis de las partes en forma reduccionista y no en sus interacciones e interconectividad. Por último, acostumbrarlo y animarlo a que adquiera el hábito de rediseñar sus saberes para que se transformen en conocimientos genuinos. Para el caso de la EA, y sobre todo en la génesis de un modelo no formal de educación ambiental para la conservación con fines de protección de los humedales Bañó y Los Negros, Covas (sf) advierte que el enfoque sistémico en la EA requiere un proyecto planteado desde una visión global que considere que se trata de un sistema abierto en el que el todo es más que la suma de sus partes, en éste es más explicativo el conocimiento de las interrelaciones, donde se busca el tratamiento interdisciplinario, se valora la estructura y funcionamiento, teniendo en cuenta aspectos dinámicos, evolutivos y la realización del sistema dada su complejidad. En síntesis, el enfoque sistémico aplicado al campo educativo, contempla la conexión entre los individuos y el contexto, tanto el inmediato, familiar, educativo, entre iguales, como el más amplio y genérico, social, político, religioso, cultural, etc., teniendo en cuenta sus interacciones recíprocas en una constante retroalimentación de comunicación (Compag, sf). 


\section{¿Por qué aprendizaje significativo, andragogía y teoría sistémica de la enseñanza como base pedagógica para el modelo?}

Los humedales de Baño y Los Negros se encuentran ubicados en el bajo Sinú, exactamente entre los corregimientos de Cotocá Arriba, Castilleral y Palo de Agua, todos pertenecientes al área rural del municipio de Lorica, departamento de Córdoba, Colombia. La primera está localizada a $09^{\circ} 08^{\prime} 09^{\prime \prime} \mathrm{N}$ y $075^{\circ} 50^{\prime} 12^{\prime \prime} \mathrm{W}$, la segunda a $09^{\circ} 07^{\prime} 59^{\prime \prime}$ N y $075^{\circ} 50^{\prime} 49^{\prime \prime}$ W. (Ver Figura 1).

\section{Figura 1. Fotografía aérea de las Ciénagas de Bañó y Los Negros.}

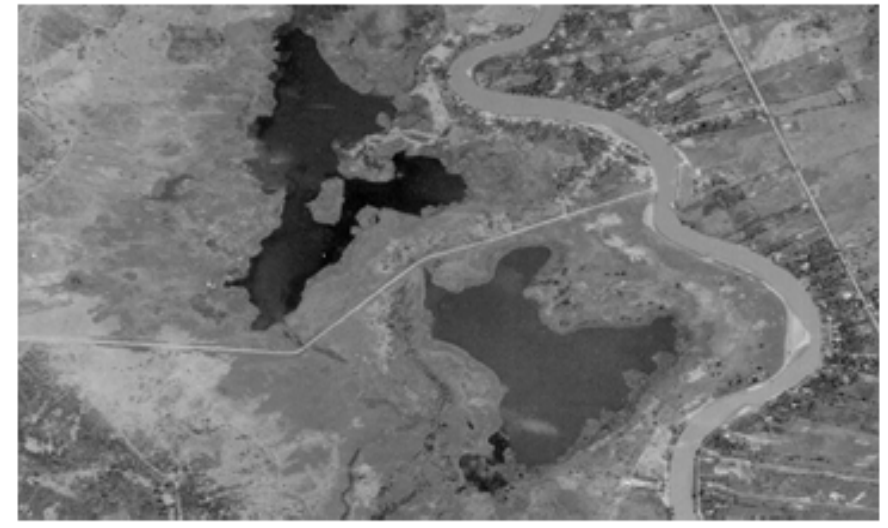

Margen izquierdo del río Sinú, en el corregimiento de Cotocá Arriba, municipio de Lorica.

Ciénaga de Bañó (parte inferior en la foto) y Ciénaga Los Negros (parte superior en la foto).

Fuente: Instituto Geográfico Agustín Codazzi (1994).

Las ciénagas de Bañó y los Negros son humedales de tipo ribereño que bordean el cauce del río Sinú. En el área de influencia de los humedales se observa una serie de presiones a las que se ven sometidos ambos espejos por parte de las comunidades vecinas. Existen problemas ambientales que van desde la desecación de los humedales, pérdida de la biodiversidad, mal manejo de los recursos suelo y agua, saneamiento básico deficiente y manejo inadecuado de residuos. Como una respuesta que ayude a mitigar la problemática ambiental por la que atraviesan estos ecosistemas, se pretende generar un modelo de educación no formal para mitigar los impactos ocasionados por los habitantes de los humedales. Se han tenido como ejes estructurantes de dicho modelo al perfil ambiental del territorio, un análisis de los factores que están incidiendo en conductas ambientales negativas de los habitantes de los humedales hacia su medio y la definición de una base pedagógica acorde con la población a trabajar.

Para el caso en particular, la población objeto de estudio oscila entre 20 y 55 años de edad, tratándose de población adulta. Por lo tanto, cuentan con conocimientos previos de su entorno debido a que tienen largo tiempo de vivir en la zona. Lo anterior se puede demostrar a partir del análisis de resultados obtenidos con la aplicación del cuestionario para la medición de las variables socioambientales, según la cual, por lo menos el $53,4 \%$ de las viviendas cuenta con un individuo que tiene más de 30 años de habitar en el territorio y un $32,4 \%$ se encuentra en un rango de 16 a 30 años de habitar en la zona. Si se suman ambos porcentajes y se tienen en cuenta los dos rangos, se estaría hablando de un $85,8 \%$ del total de la población que tiene 16 años o más de vivir en el área, lo que indica que muchos de estos individuos han nacido, crecido y 
permanecido en esta región; además de tener un conocimiento amplio y empírico de los humedales y de los problemas presentes en ellos y como éstos se han originado (Ver Gráficas 1 y 2).

Gráfica 1. Población área del estudio

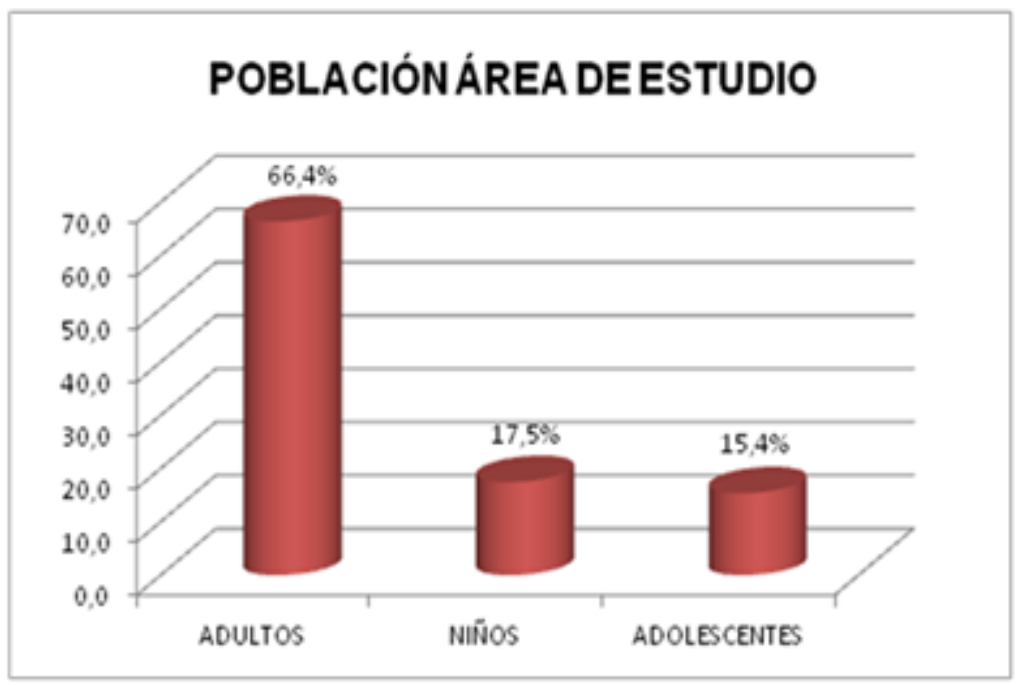

Fuente: Elaboración propia.

Gráfica 2: Tiempo de residir en la comunidad.

\section{TIEMPO DE RESIDIR EN LA COMUNIDAD}

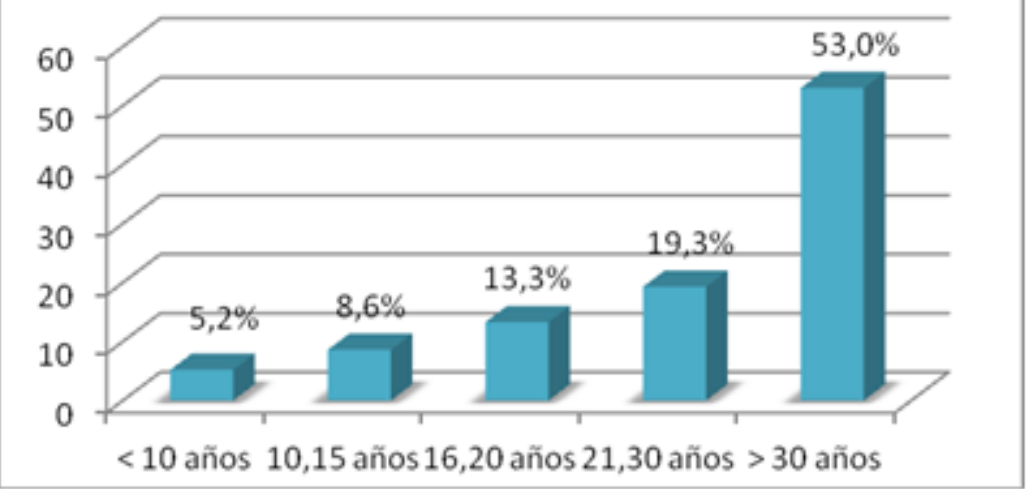

Fuente: Elaboración propia.

Como se mencionó anteriormente, los habitantes de los humedales poseen conocimientos previos de este tipo de ecosistemas, obtenidos muchas veces de forma empírica por la interacción constante con esos. Desde el aprendizaje significativo se podría utilizar ese conocimiento que ya poseen y que es relevante, un "subsunsor" en términos de Ausubel, para enlazarlo con una nueva información. Para ser más claros al respecto, un ejemplo son los habitantes de los alrededores de las ciénagas, quienes por su relación directa con los humedales poseen un conocimiento sobre lo que son los humedales. Por ende, si se les explica el concepto de ecosistema y su importancia para el planeta y a la vez lo asocian con su conocimiento previo de lo que es un humedal, aprenderán que éstos son también un tipo de ecosistema y que tiene 
relevancia para el equilibrio del planeta, lográndose un aprendizaje significativo, o por lo menos, generando el espacio para la construcción de un conocimiento bajo esta teoría pedagógica. En el mismo orden de ideas, como los espejos de agua presentan problemas ambientales, se podría por ejemplo, construir un mapa mental ${ }^{6}$, de manera individual o colectiva, a partir de una explicación de los que es un problema ambiental, las causas, efectos del mismo, los actores involucrados y las posibles soluciones que se pudieran dar a partir de las ideas planteadas por la comunidad.

Esto ayudaría a desarrollar todo el concepto de lo que es e implica un problema ambiental, a la vez de relacionarlos a los existentes en Bañó y los Negros. Podría solicitársele a un individuo que indicara un ejemplo de algún problema que estuviera afectando a los humedales, qué personas o instituciones estarían involucradas en éste y quésoluciones propondría para su mitigación (Ver ejemplo Figura 2).

Figura 2. Ejemplo de un posible mapa mental construido por un(os)

$$
\begin{aligned}
& \text { habitante(s) de las ciénagas utilizando el aprendizaje significativo } \\
& \text { como modelo de enseñanza y aprendizaje. }
\end{aligned}
$$

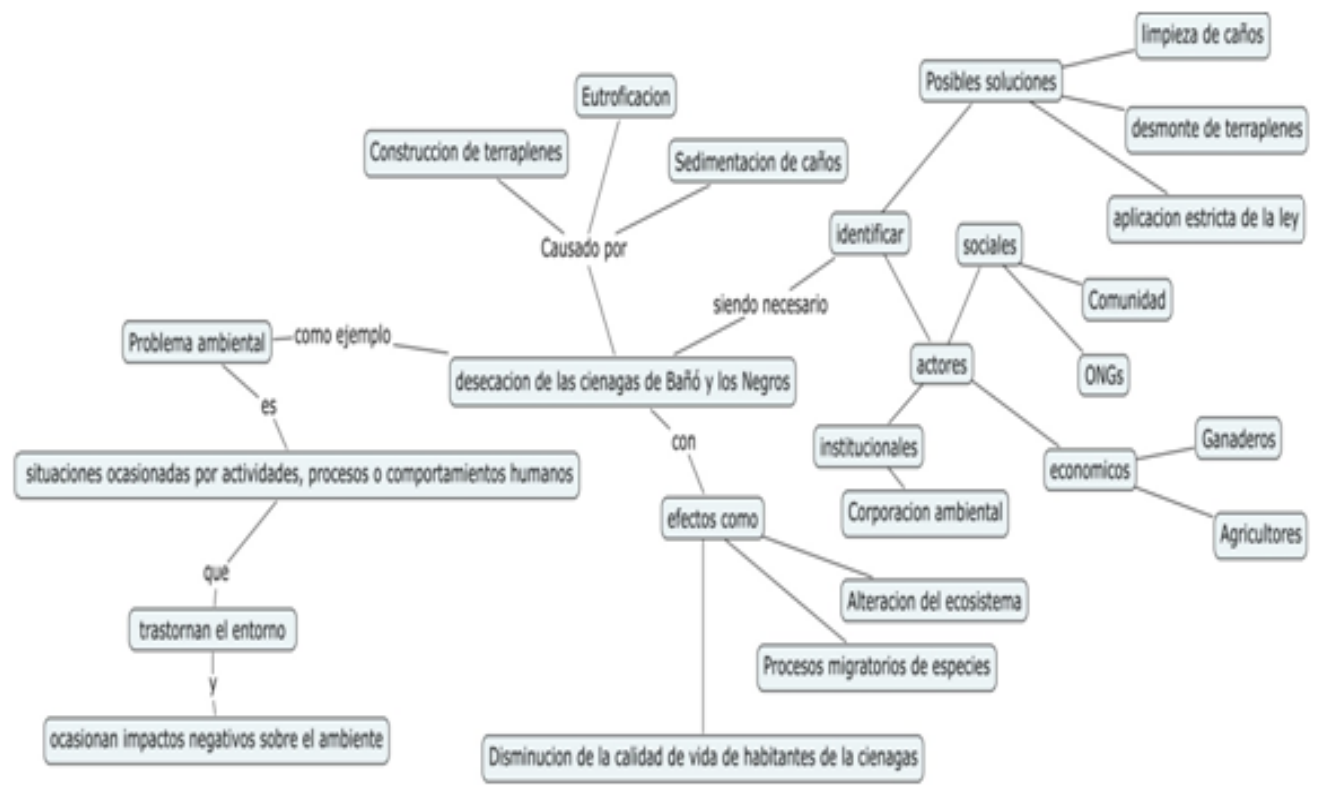

Fuente: Elaboración propia.

La educación andragógica es un elemento a considerar a la hora de generar un soporte pedagógico para trabajar con la población objeto de estudio. Los habitantes de los sectores aledaños a los humedales son personas que tienen muy bajos ingresos, poca preparación académica y fuentes de empleo, familias numerosas, necesidades básicas insatisfechas, ausencia de una cultura ambiental y poca iniciativa hacia la protección, conservación y un uso racional de los recursos naturales. Basándose en los datos estadísticos obtenidos con la aplicación del instrumento en campo, de las 645 viviendas habitadas se estimó que un 48,3\% cuenta por lo menos con un individuo que no ha desarrollado de forma adecuada sus estudios básicos (Ver Gráfica 3). 


\section{Gráfica 3. Nivel de educación}

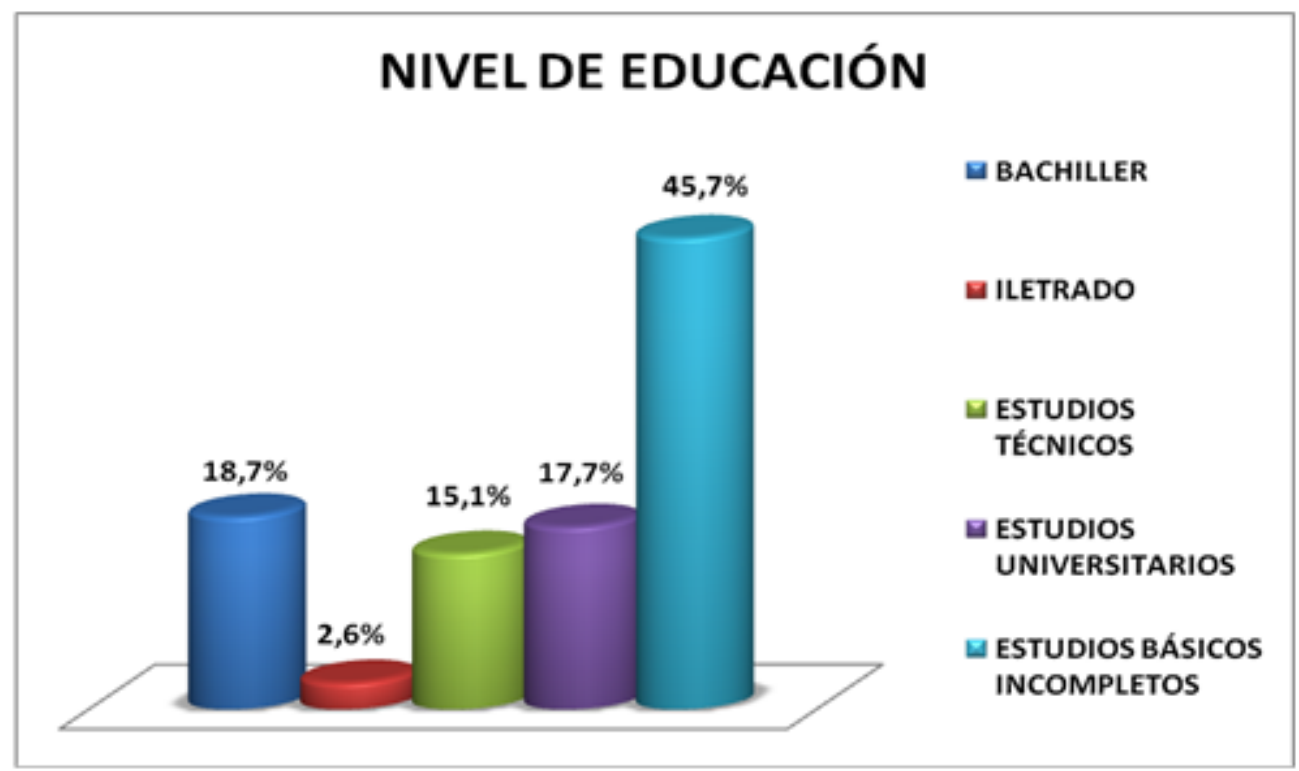

Fuente: Elaboración propia.

Un 53\% (342) de las familias en el territorio reciben ingresos diarios menores a \$ 10.000 (un poco más de US\$ 5; se podría decir que en promedio reciben entre $\$ 5000$ a $\$ 7000$ por familia) (Ver Tabla 1).

Existe un $43 \%$ de las viviendas donde por lo menos una persona no conoce el término de cultura ambiental (Ver Gráfica 4). Así mismo, un 83\% de las viviendas cuenta por lo menos con un individuo que no se siente responsable del deterioro de las ciénagas (VerGráfica 5).

Se prioriza que las comunidades sean participantes activas en el diseño e implementación del modelo con el fin de que se apropien de éste y pueda convertirse en un instrumento de desarrollo e innovación social. El modelo no formal de educación ambiental debe contribuir a concretar las potencialidades de los humedales y sus habitantes, que conlleve a generar las bases para viabilizar un desarrollo sostenible y poder utilizar de manera racional los recursos existentes.

Tabla 1. Ingresos por vivienda.

\begin{tabular}{|l|c|c|c|c|c|}
\hline \multicolumn{7}{|c|}{ INGRESOS VIVIENDA } \\
\hline PERIODICIDAD & $<\$ 10000$ & $\$ 10000, \$<20550$ & $\$ 20550, \$ 41100$ & $>\$ 40100,<\$ 61650$ & $>\$ 61650$ \\
\hline DIARIAMENTE & 163,35 & 110,67 & 0 & 0 & 8,1 \\
\hline SEMANALIENTE & 106,46 & 49,69 & 46,6 & 12,17 & 0 \\
\hline QUINCENALMENTE & 28,38 & 20,27 & 0 & 0 & 0 \\
\hline MENSUALMENTE & 44,58 & 24,31 & 0 & 33,42 & 0 \\
\hline TOTAL & 342,77 & 204,94 & 46,6 & 44,59 & 8,1 \\
\hline
\end{tabular}

Fuente: Elaboración propia. 


\section{Gráfica 4. Conocimiento del término cultura ambiental.}

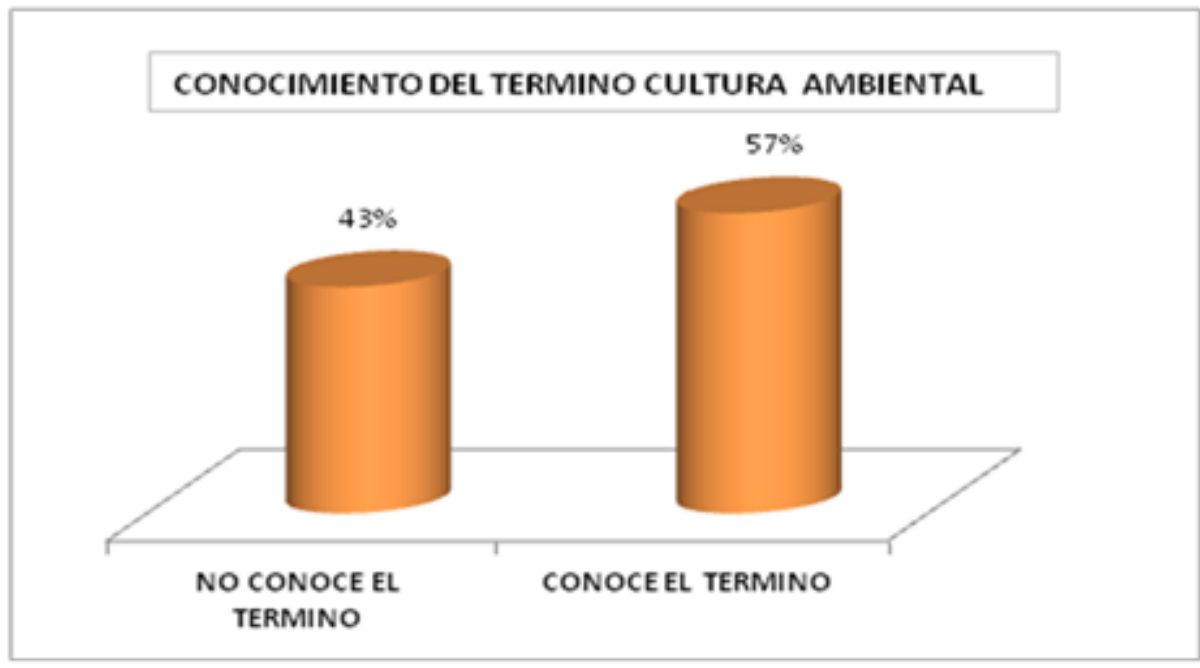

Fuente: Elaboración propia

Gráfica 5. Grado de responsabilidad frente

al deterioro de los humedales.

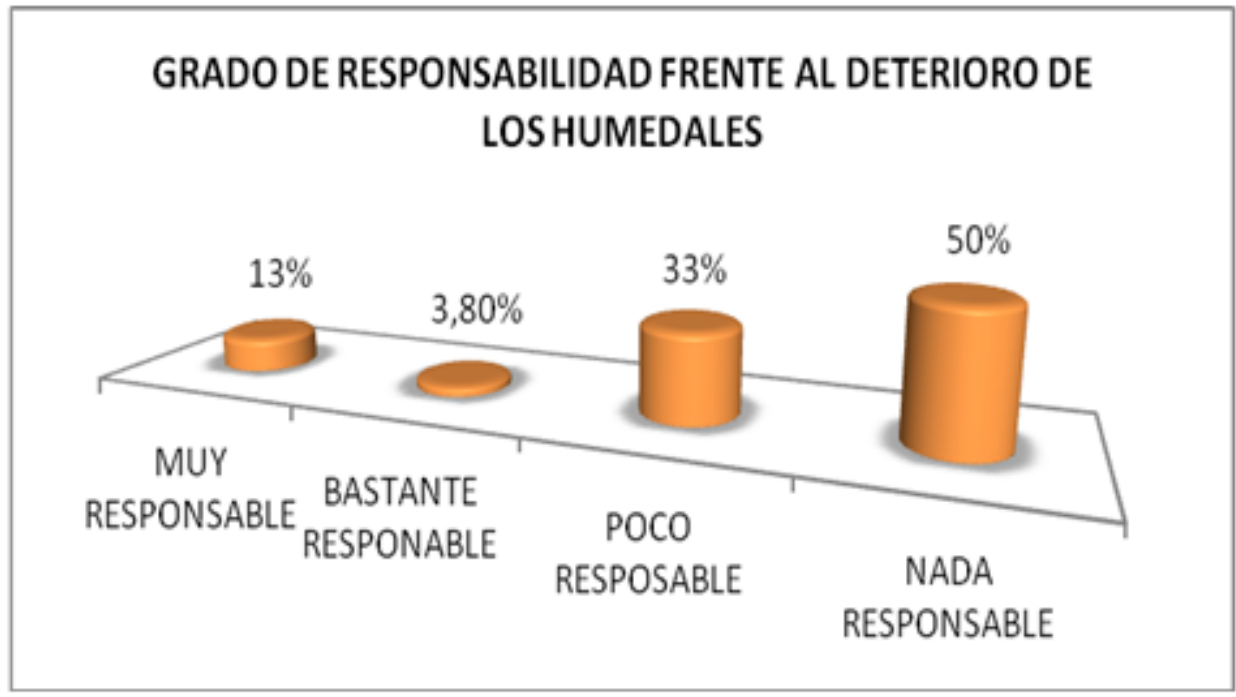

Fuente: Elaboración propia.

Ahora, desde la perspectiva de la educación andragógica, y como la población con la que se pretende trabajar es adulta, se buscaría aplicar en ésta los principios de horizontalidad y participación, es decir, desarrollar a partir del modelo educativo un proyecto de vida individual y que a su vez configure en una colectividad donde todos acuerden un mismo nivel jerárquico con el compromiso y participación de todos. Un nivel donde desde la individualidad se construya una colectividad con el fin de generar procesos de aprendizaje que contribuyan a mejorar la relación hombre-medio. La andragogía permitirá que cada uno de los individuos involucrados en el desarrollo del 
modelo valore sus experiencias propias y ajenas con el fin de construir su proyecto de vida, siendo el individuo o los individuos los únicos responsables del proceso. Así mismo, la andragogía promueve la participación como uno de sus pilares, es a través de ésta que cada individuo puede proponer o construir alternativas de solución a la problemática ambiental de los humedales, mediante la creación de redes o alianzas grupales, entre otras estrategias. Un ejemplo de lo anterior sería el desarrollo de proyectos de vida comunitaria basados en el ecoturismo, donde las comunidades participantes recibirían formación ambiental y a la vez podrían crear ingresos económicos para sus hogares a partir del diseño de paquetes eco turísticos con los que enseñen a los visitantes lo valioso de un ecosistema tipo humedal como lo son Bañó y Los Negros.

Por otra parte, el hecho que las poblaciones no se sientan responsables del deterioro ambiental de los humedales se convierte en un indicador de que no poseen una visión holística o sistémica de su territorio y ante tal deficiencia no perciben que de manera directa o indirecta sí lo son, y estarían pecando ya sea por omisión o acción. De allí, la necesidad de incluir en la base pedagógica del modelo la teoría sistémica de la educación aplicada a este contexto en particular. La teoría sistémica de la enseñanza se encuentra soportada sobre la teoría general de sistemas, la cual permite relacionar los sistemas biofísicos y los sistemas sociales hacia el logro de una integración interdisciplinaria. En la medida en que los individuos residentes de los humedales desarrollen un pensamiento sistémico, entenderán que sus acciones no son aisladas y tienen repercusiones al medio. Según Rosell (1989) el enfoque de sistema, también denominado enfoque sistémico, significa que el modo de abordar los objetos y fenómenos no puede ser aislado, sino que tienen que verse como parte de un todo. No es la suma de elementos, sino un conjunto de elementos que se encuentran en interacción, de forma integral, que produce nuevas cualidades con características diferentes, cuyo resultado es superior al de los componentes que lo forman y provocan un salto de calidad.

Una de las posibles aplicaciones que se podrían desarrollar con la teoría sistémica es tomar uno de los problemas ambientales que los afecta y realizar un análisis holístico para el mismo, poder establecer todas las causas y efectos que se deriven de este a escala local, regional, nacional y global y cómo podría solucionarlo a partir de sus aportaciones. Hay que recordar que la sistematización es una actividad mental que se efectúa en el proceso de estudio, consistente en reunir en grupos los objetos y fenómenos, según determinados rasgos o principios, y ordenar las materias según determinados sistemas, en los que al guardar cada una de sus partes ciertas relaciones con las demás, forman un conjunto armónico (Danilov et. al. 1989; citado por Rosell, 2003).

\section{Conclusiones}

A partir del análisis de teorías educativas y de los datos estadísticos obtenidos con la aplicación del instrumento de investigación tipo cuestionario, que contribuyeron a la generación de una base pedagógica que respondiera a las particularidades de las poblaciones presentes en los humedales Baño y Los Negros, se puede concluir que:

- La educación no formal para los adultos de las comunidades cercanas al área de estudio es una necesidad urgente debido: 
- $\quad$ Al bajo nivel de conciencia compartida entre los miembros de éstas con respecto a su potencial de intervenir positivamente en los procesos de organización comunitaria, para resolver los problemas de deterioro del medio ambiente en sus territorios

A los conocimientos y actitudes requeridos para lograr una praxis sostenida de gestión ambiental, que resulte en el mejoramiento de las condiciones de los humedales estudiados; y

A las escasas actividades conjuntas entre las organizaciones comunitarias y las instancias gubernamentales y no gubernamentales, en torno a la solución de los problemas ambientales de los humedales bajo estudio.

- Las características propuestas del modelo educativo no formal y participativo que se analizó en el presente estudio ofrece una buena opción para promover la organización comunitaria con respecto al desarrollo sostenible porque:

Vincula una propuesta educativa con las condiciones de mejoramiento ambiental de las comunidades del estudio;

Las comunidades estudiadas se vuelven co-partícipes en el desarrollo sostenible de sus territorios; y

Se centra la acción educativa en el mejoramiento de la situación ambiental local en donde la gestión ambiental es un esfuerzo colectivo de promover el conocimiento público que difunde conocimientos teóricos de acción comunitaria para transformar el aprendizaje en sistemas de comportamiento.

- $\quad$ La base pedagógica propuesta aquí para un modelo de educación ambiental no formal, debe tener como fin:

- $\quad$ Cambiar las actitudes de las personas que viven en situaciones de deterioro ambiental, a partir de una serie de supuestos pedagógicos que se convierten en elementos integradores de una base pedagógica, para la generación de una propuesta educativa no formal participativa para mejorar la relación ser humano-medio;

Apoyar proyectos de vida basados en los principios de igualdad y trabajo colaborativo, y un firme compromiso de aprender y propender por el desarrollo sostenible de sus territorios; y

Despertar las conciencias de los individuos participantes y a mejorar la relación de los habitantes con su medio ambiente.

\section{Recomendaciones}

Por lo señalado anteriormente, en torno a la educación no formal y participativa que se analizó en el presente documento, se hacen las siguientes sugerencias:

- Fomentar espacios de formación no formal en educación ambiental donde se desarrollen estrategias pedagógicas basadas en la andragogía, la teoría sistémica de la enseñanza y el aprendizaje significativo, en el marco de un trabajo comunitario que se articule con las políticas y líneas de trabajo propuestas en el área ambiental por parte del estado y donde la participación activa y el compromiso por parte de las comunidades rurales, estableciendo así objetivos y metas claras que faciliten alcanzar la sostenibilidad territorial desde lo individual y lo colectivo.

- Reflexionar sobre la importancia de construir una base pedagógica que responda tanto a las particularidades del territorio como a la población que se beneficie con la implementación de programas de EA no formal, en comunidades campesinas con 
población adulta, al igual que valorar los conocimientos previos de estas comunidades y a partir de estos conocimientos generar procesos formativos no formales eficientes.

- Construir con las comunidades rurales, a partir de procesos formativos no formales en educación ambiental, como también con el diseño e implementación de una base pedagógica contextualizada.

Es importante tener presente que las actitudes negativas hacia el ambiente no son siempre evidentes, que muchas veces vienen disfrazadas de buena voluntad, buenas intenciones, abnegación, resignación, comprensión, tolerancia, paciencia y hasta de amor. No todo es lo que parece en torno a las actitudes y su cambio.

\section{Referencias}

- Adam, F. (1971). Metodología andragógica. Anea idea. Caracas: Venezuela.

- Alcalá, A. (2001). La praxis andragógica en los adultos de edad avanzada. Revista Informe de Investigaciones Educativas, No. 1-2: pp. 33-47. Recuperado de: Link

- Antolin, J. (2010). El pensamiento sistémico integral enfoque de la enseñanza en el aula. Revista electrónica Hekademus, No. 8. Vol. 3. Recuperado de: Link

- Ausubel, D., Novak, P., y Hanesian, H. (1983). Psicología educativa: un punto de vista cognoscitivo. $2^{\circ}$ Ed. Trillas: México.

- Bolívar, M. (Julio, 2009) ¿Como fomentar el aprendizaje significativo en el aula? Temas para la Educación, No. 3. Recuperado de: Link

- Buenfil, B. y Rosa, N. (1991). Análisis del discurso y educación en México. CINVESTAV: México.

- Camors, J. (2009). Educación no formal política educativa del MEC 2005 - 2009. En: Ministerio de Educación y Cultura de Uruguay (MEC), Aportes para la elaboración de propuesta de políticas educativas. Montevideo, Uruguay.

- Compag, E. (sf). El modelo sistémico aplicado al campo educativo. Recuperado de:Link

- Covas, O. (sf). Educación ambiental a partir de tres enfoques: comunitario, sistémico e interdisciplinar. Recuperado de: Link

- Fernández, Néstor (2001). Andragogía: su ubicación en la educación continua. Universidad Autónoma de México: Dirección de Educación Continua.

- González, E. (1993). Hacia una estrategia nacional y plan de acción de educación ambiental. INE, UNESCO, SEDESOL: México.

- Guier, E., Rodríguez, M. y Zúñiga, M. (2002). Educación ambiental en Costa Rica: tendencias evolutivas, perspectivas y desafíos. San José: Editorial UNED. 
- Knowles, M. (1980). Práctica moderna de la educación en adultos. Temas de educación. Año1.N⒉ Caracas: Venezuela. Chicago: USA.

- Ludojoski, R. (2008). Andragogiía o educación del adulto. Ciberdocencia. Recuperado de: Link

- Moreira, M. A. (2000b). Aprendizaje significativo crítico. Atas do III Encontro Internacional de Aprendizagem Significativa. Peniche, Portugal, pp. 33-45. (Trad. Ileana Greca).

- Nemeth, A. (1991). La educación sistémica. Recuperado de: Link

- Ojeda, A., Díaz, F., Gonzales, F., Pinedo, P. y Hernández, M. (2007). Los mapas conceptuales: una poderosa herramienta para el aprendizaje significativo. Acimed No 5. Recuperado de: Link

- Pereira, M (sf). La andragogía. Nuevo paradigma educativo. Disponible en Link

- Rodríguez Palmero, M. L. (2004 a). La teoría del aprendizaje significativo. Ponencia presentada en la First Intenational Conference on Concept Mapping. Pamplona (España), 14-17 de septiembre, pp. 535-544.

- Rodríguez Palmero, M. L. (2004b). Aprendizaje significativo e interacción personal. En Moreira, M. A., Caballero Sahelices, C. y Rodríguez Palmero, M. L. Aprendizaje Significativo: Interacción personal, progresividad y lenguaje. Universidad de Burgos. Servicio de Publicaciones, pp. 15-46.

- Rodríguez Palmero, M. L. (2008). La teoría del aprendizaje significativo. En Rodríguez Palmero, M. L. (org.): La teoría del aprendizaje significativo en la perspectiva de la psicología cognitiva. (Pp. 7-45). Barcelona: Ed. Octaedro.

- Rojano, J. (2008). Conceptos básicos en pedagogía. Revista electrónica de Humanidades, Educación y Comunicación Social, No. 4.. Recuperado de: Link

- Rosell, W. (1989.) Medios de enseñanza. La Habana: Pueblo y Educación.

- Rosell, W. y Mas, M. (2003). El enfoque sistémico en el contenido de la enseñanza. Recuperado de: Link

- Torres, M., Fermín, I., Piñero, M. Arroyo, C. (1994). La praxis andragógica. La horizontalidad y la participación en la situación de aprendizaje. Mérida: Edición. Universidad de Los Andes. Consejo de Publicaciones: Venezuela.

- Trilla, J. (1996). La educación fuera de la escuela. Barcelona: Editorial Ariel. 
- Valdez, J. (2011). Andragogía y educación popular: un diálogo inminente EnComunicación, educación y ciudadanía.

- Valenzuela, A. (2004). Enfoque de sistemas aplicado a la educación. Recuperado de: Link

1. Docente investigador del programa de ingenieria sanitaria y ambiental. Universidad Pontificia Bolivariana. Seccional Montería, Departamento de Córdoba, Colombia. Cel: 3007368206. Correo electrónico: Jorge.villadiegol@upb.edu.co. orcid.org/0000-00026151-9987

2. Doctor en Pedagogía. Profesor titular del programa de Doctorado en Ciencias Naturales para el Desarrollo. Tel.: 001-595-9236497. Correo electrónico: d_huffman@yahoo.com. Instituto Tecnológico de Costa Rica. Costa Rica. orcid.org/0000-0003-0523-1046

3. Estadístico. Tel 3156199556. Correo electrónico: guerrerostalyn@hotmail.com. Fundación Salud Mental Para Todos. Departamento de Córdoba, Montería, Colombia. orcid.org/0000-0002-4351-5245

4. Máster en Gestión Ambiental. Tel 3156199556. Correo electrónico:acobo71@yahoo.com. Fundación Kontiki. Departamento de Bolívar, Cartagena de Indias, Colombia. orcid.org/0000-0001-8768-262X

5. Conjunto de acciones, actividades y tareas que al ser administradas aplicando principios y estrategias andragógicas adecuadas, sea posible facilitar el proceso de aprendizaje en el adulto (Alcalá, 2000).

6. Nota: La utilización del mapa mental podría ser una de las herramientas o técnicas que se pudiera aplicar en las comunidades a trabajar, no se quiere decir con esto que sea la única, entre tantas otras se cuenta con la mesa redonda, el debate, panel, mapas sonoros, etc. Todo dependerá de las características de la población beneficiada con el modelo a la hora de realizar su validación mediante una prueba piloto.

Para citar este artículo: Villadiego-Lorduy, J.; Huffman-Schwocho, D.; GuerreroGómez, S. y Cortecereo-Bossio, A. (2017). Base pedagógica para generar un modelo no formal de educación ambiental. Revista Luna Azul, 44, 316-333. DOl: 10.17151/luaz.2017.44.19.

Recuperado de http://200.21.104.25/lunazul/index.php/component/content/article?id=240

Esta obra está bajo una Licencia de Creative Commons Reconocimiento CC BY

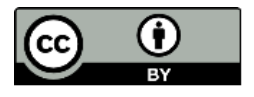

